\title{
Advanced Centralized Clustering: A Novel Protocol for Energy Efficient in Wireless Sensor Network
}

\author{
Rabiaa Elkamel ${ }^{*}$, Asma Messaoudi², Adnane Cherif ${ }^{3}$ \\ ${ }^{1}$ Innov'COM Laboratory, National Engineering School of Carthage, University Carthage, Tunisia. \\ ${ }^{2}$ InnovCOMLaboratory, National Engineering School of Tunis,University Tunis El Manar, Tunisia. \\ ${ }^{3}$ Innov'COM Laboratory, Faculty of Science of Tunis, University Tunis El Manar, Tunisia. \\ * Corresponding author. Tel.: +21693042456; email: elkamelrabiaa@gmail.com \\ Manuscript submitted October 31, 2017; accepted January 25, 2018. \\ doi: 10.17706/jcp.13.8.948-954
}

\begin{abstract}
Energy conservation is a key issue in Wireless Sensor Networks based systems design. Clustering based routing protocols have been developed in orderto reduce network traffic to the BS and thus to extend the network lifetime. A clustering variantis LEACH which is one of the most prominent hierarchical routing protocols used in wireless sensor networks(WSN). Maximizing the network lifetime is one of the major challenges. Maximizing a sensor network lifetime is equivalent to minimizing the different energy sources. In fact, a sensor node consumes energy to accomplish.In fact, a sensor node consumes energy to accomplishhis roles in the networkwhich are mainly processing and transmission of a set of physical quantity on the environment around it. This paper proposes a hierarchical routing protocol based on LEACH but the difference lies in the mode of clusters formation and clusters heads selection $(\mathrm{CH})$ ensuring balance between energy consumption sensors. Simulations show that the proposed protocol extends the life of the network compared to LEACH and iMod-leach.
\end{abstract}

Key words: WSN, hierarchical, clustering, maximizing lifetime, energy efficiency, performances.

\section{Introduction}

Sensor networks have a very wide application, whether in science, logistics, military or health. According to MIT's Technology Review, it is one of the ten new technologiesthat will shake the world and our way of life and work [1]. A sensor is a mini component used for performing a certain treatment, collecting sensory information and communicating with other nodes connected to the network. It is not integrated with any existing communication architecture, but it communicates using an ad hoc wireless network.It is difficult to integrate it due to certain constraints: consumption energy, memory space, etc. [2]. The electrical supply is provided by a single battery; both information processing and communication consumption calculations should be optimized [3]. Nodes deployment is either deterministic (manual, robots) or random (aircraft, missile). A sensor ensures not only data acquisition and processing but also communication.The latter task is the most energy intensive one. A good energy management scheme must, as a priority, consider the communications. Routing protocols are responsible of the discovery and maintenance of paths in the network. One of the main design objectives of sensor networks is to provide a data communication while trying to extend the network lifetime and to prevent connectivity degradation using aggressive power management techniques. The routing protocols designin the sensor network is influenced by many difficult issues such as fault tolerance and physical constraints. These factors must be overcome through 
development of clever routing protocols. The space design for the sensor network routing algorithms is wide enough. Taking into account procedures, routing protocols can be coarsely classified into the following two criteria: the network topology and communication paradigms [3], [4]. Thus, in recent years the research has focused on routing protocols to convey the captured information to the BS while consuming less energy and extending the WSN life. The adopted solution to organize a large WSN is to group nodes into clusters. In this type of communication based organization,an intra-cluster and inter-cluster routing reduces the number of participatingnodesin over long distancescommunications. Each node cluster is identified by a leader, called cluster manager or cluster-head, which coordinate its group's activities such as data routing, aggregation, synchronization, etc. Many clustering protocols have been proposed in the scientific literature such asPEGASIS [5] TEEN [6] and imodeLEACH [7].

Proposed by Heinzelman et al., LEACH [8] is one of the most popular routing protocols to WSN. It is a hierarchical routing protocol based on cluster formation (group).

This paper proposes an improvement of LEACH with better performance in terms of power consumption and sensor networks lifetime. Section 2 presents two routing protocols based clustering. Section 3 described the proposed protocol. Section 4 presents the simulations results. Finally, Section 5 contains conclusions of this work and presents the future work.

\section{LEACH}

The goal of routing protocol is to route information from a source to a destination effectively [9]. The major constraint in routing in WSN is to route the information captured by minimizing the power consumption of the nodes.Several routing protocols have been proposed in the literature. In this section the existing representative cluster-based routing protocols proposed for WSN including LEACH , LEACH-C [10].

LEACH is considered the first hierarchical routing protocol based on the approach based on the groups. It combines efficient consumption energy and the quality of access to the media, and this is based on the division into groups, a view to enable the use of the concept of data aggregation for better performance in terms of lifetime. The communication architecture of LEACH consists of, similar to cellular networks, to form cells based on the amplitude of the signal, and to use the cell headers as routers to the sink. These cells are called groups (clusters) and the heads are the group leaders (cluster-heads). The group leaders are randomly chosen in a specific election algorithm based on a probability function that takes into account different criteria are based the percentage of desired $\mathrm{CHs}$ and the number of iterations during which a node took the role of $\mathrm{CH}$. The nodes are responsible for collecting the data, sent to their $\mathrm{CH}$ which the aggregate and transmit the aggregation results sink in a unicast communication (only one hop). The protocol is composed by rounds, in which a round is divided into set-up phase and steady phase.Into set-up phase the nodes self-elect for the CHs. They are based on the desired percentage of CHs and the number of iterations during which a node took the role of $\mathrm{CH}$. Thus, a node $\mathrm{n}$ is a random value between 0 and 1 . If the value is less than the threshold T (n), the node declares CH. CHS inform their neighbors of their election. Each remaining node decides to choose the nearest $\mathrm{CH}$. After the formation of clusters, each $\mathrm{CH}$ programs the nodes belonging to the cluster in their sending the codes and the communication frequencies. Each node other than $\mathrm{CH}$, turned off his antenna when not communicating his data. LEACH precise, also, a data aggregation level $\mathrm{CHs}$ for more energy conservation. However, many critics are made to the protocol LEACH to know: The rotation of the role of $\mathrm{CH}$ on all nodes of the cluster, makes it possible firstly to balance the energy consumption of the cluster. But, it generates a consumption of energy, as each rotation $\mathrm{CH}$ requires diffusion phase to make known the new $\mathrm{CH}$, LEACH does not guarantee a homogeneous distribution of CHs on the network, because the only $\mathrm{CH}$ election criterion is a random probability. This does not prevent a CHs concentration in a limited area to the detriment of the whole network. 
To avoid these problems, the authors propose an extension of the algorithm. They proposed a centralized algorithm(LEACH-C). This is an iterative algorithm, wherein the structure of the clusters is calculated at the base station by using the optimization method of the "Simulated annealing" [11]. However, the base station assigns each iteration roles for different nodes of the network ( $\mathrm{CH}$ or single sensor). Then, operation continues in the same way as LEACH. Another extension is proposed [12]. It is the same principle that iMOD-LEACH . The principle of these protocols is introduced a soft threshold and hard threshold parameters and assigned them a fixed value. However, this algorithm does not take into account the main features of structures of WSNs is the grouping the nodes.

\section{The Proposed Protocol}

Clustering nodes is one of the techniques that can increase the lifetime of the entire network by aggregating data to the cluster head. previous works [13]-[16] have proposed, an original algorithm heuristic k-means to construct disjoint clusters to reinforce the robustness of the network, and address specific issues, namely the inter-cluster trans-mission, localization nodes and time synchronization.

The K-means algorithm is an Automatic clustering algorithm proposed by Geon [17]. From a number of initial partitions, seeking to improve iteratively partitioning. Each score is represented by a kernel ' $k$ '. The proposed scheme uses K-means algorithm which forms the clusters of objects based on the Euclidean distances between them. The pro-posed CH selection scheme consists of two steps as follows:

- Randomly choose $\mathrm{k}$ data items from X as initial centroids

- Repeat: Assign each data point to the cluster which has the closest centroid. Calculate new cluster centroids until the convergence criteria is met.

Algorithm1: k-means algorithm

Require: cluster centroid $\mu_{1}, \mu_{2, . .,} \mu_{k} \in I R^{n}$

Repeat until converge: for every $i$, set

$$
c_{i}=\arg \min \left\|x^{i}-\mu_{\mathrm{j}}\right\|^{2}
$$

for every $j$, set

$$
c_{i}=\frac{\sum_{i=1}^{m} 1\left(c^{i}=j\right) x^{i}}{\sum_{i=1}^{m} 1\left(c^{i}=j\right)}
$$

The proposed protocol involves three phases:

1. Phase of formation that we have described, clusters are formed by the k-means method. the k-means algorithm is a good method of grouping that ensures:

- A large intra-group similarity.

- A low similarity inter-group.

- Relatively efficient: $O(\mathrm{tkn})$, where $n$ is numbers of nodes, $k$ is numbers of clusters, and $t$ is iterations. Normally, $k, t<<$.

- Tending to reduce

- $E=\sum_{i=1}^{k} \sum_{p \in C_{i}}\left|p-m_{i}\right|$, with $C_{i}$ is cluster $i$ and $M_{i}$ is center of cluster $i$

2. In the second stage, a $\mathrm{CH}$ is elected in each cluster in the first round the centroid are proposed as a cluster head in the other round we will used an effective technique that is the Gaussian elimination algorithm his is done by taking account of the residual energy of the estimated future nodes, and 
the number of revolutions which may be responsible for the cluster formation to maximize network lifetime. It models the net-work and the energy expended by the nodes as a linear system and this using the Gaussian elimination algorithm to select the CHs [18]. The matrix represents the energy consumption of each cluster node. The elements $a_{i}, j$ refers to the energy consumed by the node $i j$ if the node is the $\mathrm{CH}$. Furthermore, $b_{i}$ denotes the residual energy of node $i$, and $x_{i}$ expresses the time during which the node $i$ is $\mathrm{CH}$.

3. In the third step, the CHs notify aggregateddata directly to the base station. The MAC protocol used to ensure equitable accessand without errors are CDMA (Code DivisionMultiple Access) using a code for each cluster,TDMA (Time Division Multiple Access) allocatingto each cluster node a specific time (calledFrame) to send its data.

- Nodes in a cluster, sends their data according toTDMA schedule, and cluster head receives, and aggregates the data.

- Cluster heads will send their data directlyto the base station.The flow chart of the overall operation with the proposed scheme is shown in Fig. 2.

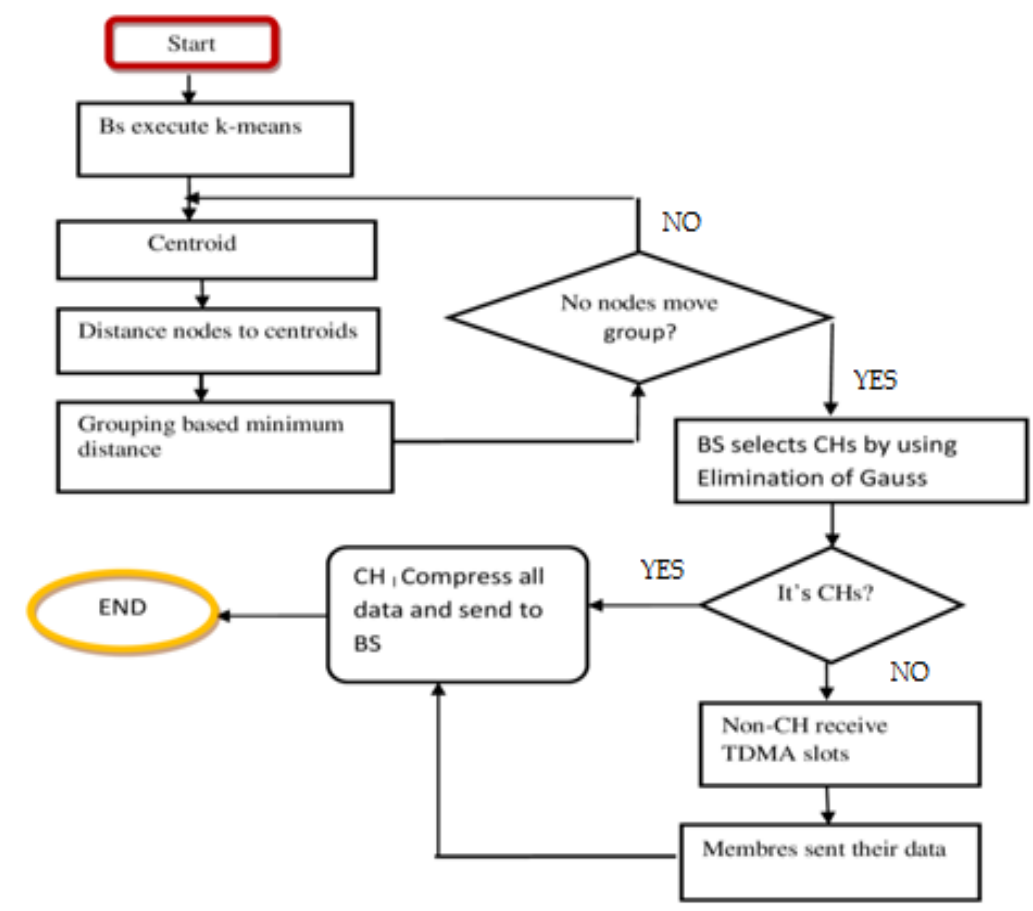

Fig. 2. Chart flow of proposed protocol.

\section{Simulation Experiments and Results}

The proposed algorithms are evaluated by simulating a 100-node network using Matlab. The sensor nodes are randomly dispersed in an area of $(100 \times 100) \mathrm{m}^{2}$; the base station is located at coordinates $(50$, 150). Each sensor node transmits a message 1000 bits. The initial energy of each sensor node is 0.1 joules. Table 1 summarizes all the simulation parameters.

Fig. 3 represents the average energy of the network function of the number of iterations for the algorithms leach, iMod-leach and Proposed Protocol. The figure below presents the rate of residual energy at the nodes. The figure shows that the average energy of nodes with the iMod-leach protocol is less than that measured with the suggestion and this by a rate of $0.14 \mathrm{~J}$ and $0.27 \mathrm{~J}$ in the round 80 . In LEACH the nearest nodes of the base station is used to carry a great number of messages to the base station. So these nodes are going to die rapidly, causing the energy required to get the remaining data to the base station to increase and more nodes to die. 
Table 1. The Arrangement of Channels

\begin{tabular}{l|c}
\hline Parameters & Values \\
\hline Area size & $(100 X 100) \mathrm{m} 2$ \\
\hline Numbers of nodes & 100 \\
\hline Initial Energy & $0.1 \mathrm{~J}$ \\
\hline Packet size & $100 \mathrm{bits}$ \\
\hline Efs & $100 \mathrm{pJ} / \mathrm{bit} / \mathrm{m} 2$ \\
\hline Eelec & $50 \mathrm{~nJ} / \mathrm{bit}$ \\
\hline Emp & $0.0013 \mathrm{pJ} / \mathrm{bit} / \mathrm{m} 4$ \\
\hline EDA & $5 \mathrm{~nJ} / \mathrm{bit}$ \\
\hline
\end{tabular}

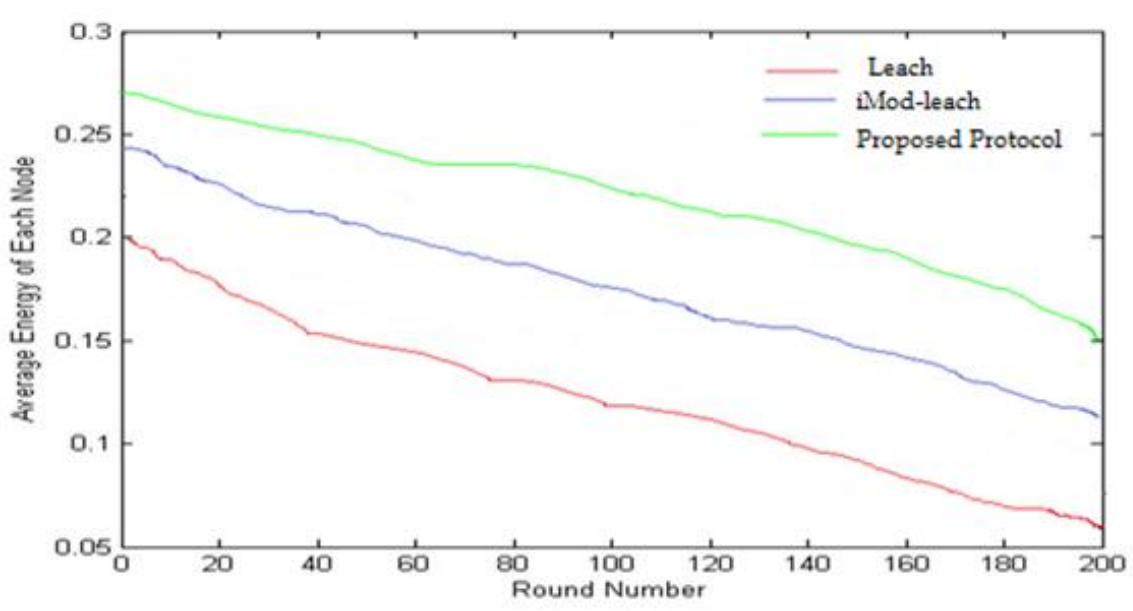

Fig. 3. Average energy of network per round.

Fig. 4 represents the number of alive nodes of the net-work function of the number of iterations for the algorithms leach, iMod-leach, "Proposed Protocol. We note that the lifetime of the network in the proposed protocol is much better than in other protocols.This is due to the fact that the energyconsumption is balanced around the nodes in the same cluster.

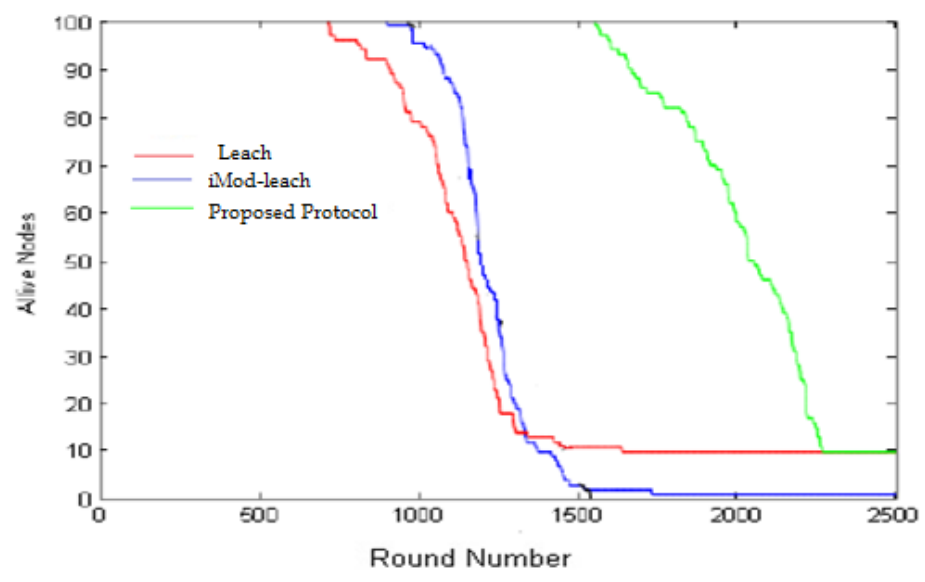

Fig. 4. Number of alive nodes per round. 


\section{Conclusion}

In wireless sensor networks, the concept of clustering is effective particularly when confronted with the problem of energy consumption. clusters based routing protocols allow sensor nodes to effectively move the sensed data to the base station. Cluster heads are sometimes chosen as specialized nodes that are less energy constrained. It is in this context that we propose a new routing protocol dedicated to wireless sensor networks; it is based on clustering using the principle of clusters formation namely algorihm of kmeans and the algorithm Gauss for selecting CH. Simulation results show that the proposed protocol extends the network lifetime compared to leach, and other protocols.

The proposed approach can be further improved by the use of certain intelligent algorithms such as genetic algorithms or ant colonies, to study the influence of the position of the base station, the application of a super-clustering on the firstlevel $\mathrm{CHs}$, and the spreading of clustering on several levels.

\section{References}

[1] 10 emerging technologies that will change the world technology review February 2003. Retrieved from http://www.technologyreview.com

[2] Engel, A., Koch, A., \& Siebel, T. (2015). A heterogeneous system architec-ture for low-power wireless sensor nodes in compute-intensive distributed applications, Clearwater Beach.

[3] Pottie, G. J., \& Kaiser, W. J. (2000). Wireless integrated network sensors. Communications of the ACM, 43(5), 551558.

[4] Davis, A., \& Chang, H. (2012). A survey of wireless sensor net-work architectures. International Journal of Computer Sci-ence and Engineering Survey (IJCSES), 3(6).

[5] Lindsey, S., \& Raghavendra, C. S. (2002). PEGASIS: Power-efficient gath-ering in sensor information systems. Proceedings of IEEE Aerospace Conference (p. 11251130).

[6] Manjeshwar, A., \& Agrawal, D. P. (2001). TEEN: A routing protocol for enhanced efficiency in wireless sensor networks. IPDPS, 189.

[7] Ahmed, S., Sandhu, M., Amjad, N., Haider, A., Akbar, M., Ah-mad, A., Khan, Z. A., Qasim, U., \& Javaid, N. iMOD LEACH: Improved MODified LEACH protocol for wireless sensor networks.

[8] Heinzelman, W. R., Chandrakasan, A., \& Balakrishnan, H. (2000). Energy-efficient communication protocol for wireless microsensor net-works. Proceedings of the 33rd Annual Hawaii International Conference on System Sciences (p. 10).

[9] Heinzelman, W., Chandrakasan, A., \& Balakrishnan, H. (2000). Energy-efficient communication protocol for wireless microsensor networks. HICSS 2000, 2.

[10] Mahmood, D., Javaid, N., Mehmood, S., Qureshi, S., Memon, A. M., \& Zaman, T. (2013). MODLEACH: A Variant of LEACH for WSNs 26th IEEE Canadian Conference on Electrical and Computer Engineering.

[11] Hannes, F., Rhrup, S., \& Stojmenovi, I. (2009). "Routing in wireless sensor networks," guide to wireless sensor networks. Springer London, 81-111.

[12] Heinzelman, W., Chandrakasan, A. P., \& Balakrishnan, H. (2002). An application-specific protocol architecture for wireless microsensor networks. IEEE Transactions on Wireless Communications, 1(4), 660-670.

[13] Mechta, D., Harous, S., \& Alem, I. (2014). Khebbab LEACH-CKM: Low energy adaptive clustering hierarchy protocol with K-means and MTE. IEEE.

[14] Harb, H., Makhoul, A., Laiymani, D., Jaber, A. \& Tawil, R. (2014). K-Means based clustering approach for data aggregation in periodic sensor networks. Proceedings of IEEE 10th International Conference on Wire-Less and Mobile Computing, Networking and Communications (WiMob).

[15] Sasikumar, P. \& SibaramKhara. K-MEANS clustering in wireless sensor networks. Proceedings of 2012 
4th International Con-ference on Computational Intelligence and Communication Net-works.

[16] Yong Park, G., Kim, H., Jeong, H. W., \& Youn, H. Y. (2013). A novel cluster head selection method based on k-means algorithm for energy efficient wireless sensor network. Proceedings of the 27th International Conference on Advanced Information Networking and Applications Work-Shops.

[17] Rakhlin, A., \& Caponnetto, A. Stability of K-Means Clustering.

[18] Nikolidakis, S. A. D., Kandris, D. Vergados, D., \& Douligeris, C. (2013). Energy efficient routing in wireless sensor networks through balanced clustering. Algorithms, 6, 29-42.

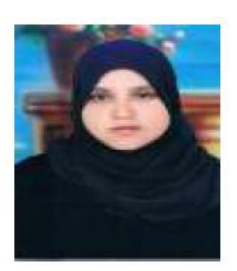

Rabiaa Elkamel obtained her fundamental license in computer science degree at 2010 and master in telecommunicationat 2014 from the Engineering Faculty of Sciences Bizerte in Tunisia. She is a researcher member of the Innov'COM Laboratoryand preparing her doctorate thesis.

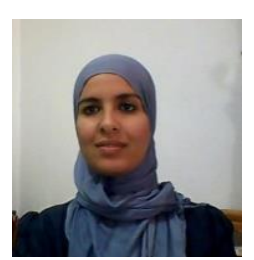

Asma Messaoudi received the engineering degree in telecommunications from the National School of engineers of Tunis (ENIT- Tunisia) June 2007. She received her master degree in communication systems from the same school on July 2008. She is a Ph.D student in Innov'COM laboratory.

Cherif Adnen obtained his engineering diploma in 1988 from the Engineering Faculty of Tunis and his Ph.D in electrical engineering and electronics in 1997. Actually he is a professorat the Science Faculty of Tunis, responsible for the signal processing laboratory. He participated in several research andcooperation projects, and is the author of more than 60 internationalcommunications and publications.. 